ÉTUDES

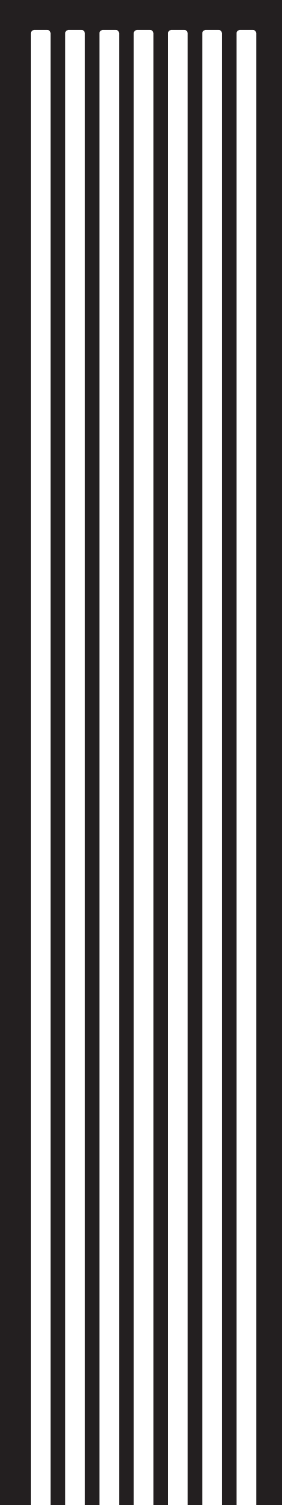




\title{
Le personnage de la vieille duchesse dans l'univers théâtral de Jean Anouilh
}

\section{Character of Old Duchess in the Theatrical Universe of Jean Anouilh}

MARIE Voždová [marie.vozdova@upol.cz]

Univerzita Palackého v Olomouci, République tchèque

\section{RÉSUMÉ}

L'article se concentre sur le personnage de la vieille duchesse dans l'univers théâtral du dramaturge français Jean Anouilh. On examine la présence de cette hérö̈ne anouilhienne dans les pièces Humulus le Muet, L'Hermine, Le Voyageur sans bagage, Le Bal des voleurs, Léocadia et L'Invitation au château. Comme ce type de personnage anouilhien reste à part de l'intérêt plus détaillé de la critique, on veut, à partir des analyses des textes, étudier ses apparitions successives, définir son caractère et montrer en quoi consiste son rôle dans la vision du monde de l'auteur.

\section{MOTS-CLÉS}

Jean Anouilh ; personnage ; duchesse ; Humulus le Muet ; L'Hermine; Le Voyageur sans bagage ; Le Bal des voleurs; Léocadia; L'Invitation au château

\begin{abstract}
The paper focuses on the character of old duchess in the theatrical universe of a French playwright Jean Anouilh. In the works Humulus le Muet, L'Hermine, Le Voyageur sans baggage, Le Bal des voleurs, Léocadia and L'Invitation au château, we analyse the presence of such a heroine. Since this type of Anouilh's characters seems to be rather marginalized by the literary criticism, our aim is to analyse these texts and on its basis subsequently explore their successive appearances, define their character and demonstrate the role they play in the author's vision of the world.
\end{abstract}

\section{KEYWORDS}

Jean Anouilh; character; duchess; Humulus le Muet; L'Hermine; Le Voyageur sans baggage; Le Bal des voleurs; Léocadia; L'Invitation au château

REÇU 2019-04-23; ACCEPTE 2019-07-07

Cet article a été écrit avec le soutien financier de MŠMT ČR attribué à UP Olomouc (IGA_FF_2019_029). 
Jean Anouilh a écrit une quarantaine de pièces de théâtre et son abondante activité créatrice couvre un demi-siècle, des années trente aux années quatre-vingt. Il a connu la guerre et l'occupation, la libération et le trouble de l'épuration où des écrivains se sont chargés de condamner d'autres écrivains, et ensuite les années de triomphe de la civilisation matérielle. Avec le temps, et sous l'influence des événements, son approche de la matière théâtrale change et sa philosophie évolue. ${ }^{1}$ Il en est de même pour la place qu'il accorde à ses protagonistes et à ses héros favoris. Dans les pièces de sa première période créatrice, le jeune héros ou la jeune héroïne idéalistes, purs, non corrompus par le monde qui les entoure, sont au centre de son théâtre. Ces héros cherchent le bonheur absolu et, s'ils ne peuvent pas accéder à leur idéal ou s'il leur est impossible de le conserver, ils préfèrent quitter ce monde. Leur destin théâtral correspond à la pensée et au sentiment de la vie du jeune Anouilh. Avec la Seconde Guerre mondiale, le dramaturge mûrit, et sa triste expérience de la guerre change sa conception des personnages et change donc son théâtre. Au centre, ce sont maintenant les adultes qui ont préféré vivre dans la société qui les entoure et ont accepté les compromissions qui leur permettent d'exister. Chacun de ces personnages, jeune ou adulte, porte la pensée ou le sentiment d'Anouilh. Outre de jeunes idéalistes intransigeants, ou des adultes qui ont vécu et sont devenus pessimistes, l'auteur met en scène le personnage récurrent de la vieille duchesse. Cette héroïne, quelque peu oubliée par la critique, est une sorte de porte-parole insolite de l'auteur. C'est au repérage de ses apparitions successives et à l'examen de son caractère que cette étude est consacrée.

\section{La Duchesse de Brignoc dans L'Humulus le muet : entre le pathétique et le grotesque}

La vieille duchesse, de la famille des Brignoc, apparaît pour la première fois en 1929 dans Humulus le Muet, une œuvre d'une dizaine de pages, divisée en quatre courtes scènes et écrite par un tout jeune auteur de dix-neuf ans dont on peut deviner la future voie théâtrale. Anouilh invente alors l'histoire extravagante du jeune Humulus, né muet mais qui, par les bons soins d'un médecin anglais, peut prononcer un seul mot par jour. Il peut aussi économiser son mot quotidien et le réserver pour un autre jour, et accumuler ainsi, par le silence, une somme de mots. Humulus tombe amoureux d'une jeune fille, Hélène, et s'abstient de parler pour économiser les mots nécessaires à son aveu. Quand, après un mois de silence, il a accumulé sa provision de mots et peut déclarer son amour, il découvre qu'Hélène est sourde et n'a rien entendu.

La pièce porte le nom du héros, Humulus le Muet, mais en réalité le jeune auteur paraît fasciné par la Duchesse, au point de lui accorder un rôle essentiel. La Duchesse apparaît dans les deux premières scènes, puis disparaît. Le personnage est important, car c'est lui qui met en tension le

1 Selon Christophe Mercier, « jusqu’à la guerre, Anouilh est une sorte de romantique, de pessimiste sentimental, mais toujours fleur bleue. Il reste un idéaliste et son théâtre a les faiblesses d’un théâtre post-adolescent. Après la guerre, plus désabusé, plus cynique, il va inventer un théâtre sec, dru, sans pitié et sans mollesse. » (Mercier 1995 : 78 ) Et il ajoute plus loin : « La légèreté provocante d'Anouilh, son mépris affiché pour toutes les théories, son désir de vivre dans la discrétion et selon son bon plaisir, correspondent à l'image qu’ont donnée à l'époque ses amis littéraires, ces « anarchistes de droite ", les Laurent, les Aymé, les Blondin, violemment opposés, au nom du droit à l'individualisme, à toutes les bannières. Anouilh, au théâtre, est une sorte de « hussard », pour reprendre le terme inventé par Bernard Frank [...] il a une morale esthétique, mais cette morale doit être exprimée à travers l'œuvre elle-même, et non pas à côté. » (Mercier 1995 : 101-102) 
mécanisme de la pièce et en fait, comme l'écrit Christophe Mercier, une «tragédie du langage à la Ionesco » (Mercier 1995 : 185). Dans les didascalies, elle est décrite ainsi : " La Duchesse, sorte de personnage fabuleux sur un immense fauteuil à oreilles armoriées. » (Anouilh 1958 : 9). On n’apprend plus rien de son physique ni de son caractère. Ce dernier se dévoile dans ses discours et dans les dialogues. Le jeune auteur crée ainsi un sorte d'archétype de son personnage, celui de la vieille femme riche aux allures fabuleuses, qui rassemble autour d'elle toute la famille dont elle devient la reine. Les objets, par exemple l'« immense fauteuil à oreilles armoriées » qui suggère le trône, font la démonstration de son pouvoir absolu ; les gens eux-mêmes sont des accessoires qui l'accompagnent et l'entourent, ils jouent pour elle le rôle de décor vivant, que ce soient les domestiques, ou l'oncle Hector, ou encore Humulus lui-même. Tout doit marcher selon ses instructions et suivre sa volonté.

La Duchesse de Brignoc se montre intraitable pour son petit-fils comme pour les domestiques. Elle est habituée à l'obéissance du personnel, du gouverneur de l'enfant et de la famille. Elle a résolu que c'est elle qui doit jouir du mot quotidien d'Humulus et se le réserve. Humulus obéit en tout à la Duchesse, malgré son anxiété d'avoir à prononcer son mot devant tout le monde. Elle explique à Hector :

Pour dire « Fors l'honneur », la devise des Brignoc, mon cher Hector, il est obligé de rester trois jours sans parler... Je fais ce petit sacrifice tous les ans au moment de la fête du pays, mais je ne saurais me passer bien longtemps d'entendre dire son mot. Car, je l'ai exigé, Humulus vient me dire son mot chaque matin. Hier, vendredi, par exemple, il m'a dit «morue». (Anouilh 1958: 10)²

L'image de la Duchesse est marquée par le comique manifeste de ses entrées et sorties, toujours liées à celle de tous ses domestiques, comme parodie d'un roi donnant audition en son palais. Son discours est fait d'ordres et de questions, composé de phrases exclamatives, mais elle prononce aussi un discours solennel, toujours le même d'une année sur l'autre, à l'occasion de la nouvelle année :

Monsieur mon petit-fils, avant même que vous lui offriez vos souhaits, votre grand-mère veut vous dire sa tendresse au seuil de cette nouvelle année. Depuis que votre pauvre mère est morte, Humulus, c'est moi qui vous aime. Vous avez une grand-mère, il faut en profiter pour l'écouter. Soyez bon et vaillant comme un vrai Brignoc. Chérissez-moi et ne m'en veuillez pas si je ne vous vois qu'aux grandes fêtes. Tout mon temps est pris par mes pauvres. (Anouilh 1958 : 11)

Quoi qu'il en soit de ses déclarations, «c'est moi qui vous aime», elle parait manquer de sentiment pour Humulus et pour son son prochain en général et, sinon pour le mot du matin, ne s'en occupe guère au prétexte que tout son temps est pris par le soin des pauvres. Et quand Humulus refuse de prononcer son mot, elle " pousse un cri terrible et s'évanouit » (Anouilh 1958 : 15).

2 La cocasserie de la situation sous le sérieux de la parole de la Duchesse, se double d'une discrète inconvenance de langage, qui échappe à la Duchesse, mais non au public populaire, "Humulus vient me dire son mot, chaque matin ", comme un petit enfant vient présenter son pot, et " morue » est certes le poisson du vendredi mais aussi insulte pour une vieille prostituée! Quand Humulus laisse tomber son bouquet, et remercie gentiment le domestique pour son aide, perdant ainsi son mot du jour, elle est prise de colère. 
Avec sa Duchesse Anouilh introduit un personnage qui, dans le monde du théâtre, se fait l'égal parodique de Dieu et il est vrai que tout le monde agit d'après son arbitre, respecte ses ordres et vit dans la crainte. Pourtant Humulus le Muet, objet déclaré de son amour, dont elle dicte la parole, devient son unique opposant, car son amour pour Hélène lui donne du courage et le pousse à se dresser contre le rôle établi. Discrètement, sous un travestissement extravagant, Anouilh met en scène une révolte du personnage le plus humble, et amoureux, Humulus, contre la Duchesse, image comique de l'Auteur tout puissant, mais qui en réalité ne l'est guère. Les obsessions du jeune Anouilh, son athéisme théâtral et religieux, et ses interrogations sur le personnage en rupture de ban avec l'anecdote convenue sont clairement mis en scène dans cette œuvre fondatrice aux allures de théâtre bouffe.

\section{La Duchesse de Granat dans L'Hermine : malédiction du pouvoir et de l'argent}

La Duchesse réapparaît dans L'Hermine, de 1931, mais pour être mise à mort par un meurtre abominable. Anouilh a écrit la pièce alors qu'il était «obsédé par [sa] maladresse à vivre, [sa] pauvreté - et surtout, il faut bien le dire, par Raskolnikov» (Anouilh 1987 : 61), comme une variation de Crime et châtiment de Dostö̈evski. L'auteur raconte l'histoire de l'amour impossible de Monime, la nièce de la Duchesse, et de Frantz, son protégé et fils de son ancien médecin décédé, que Frantz croit accomplir par le meurtre de la Duchesse.

La Duchesse de Granat ne manifeste aucun sentiment, elle paraît dépourvue de toute humanité. La force de l'argent la rend puissante, dominatrice, insensible et insupportable pour son entourage. Sa position sociale lui fait croire qu'elle peut disposer du bonheur des autres, diriger leur vies et décider pour eux. Ainsi, elle brise le bonheur des jeunes amoureux, préparant pour chacun d'eux un autre destin que celui auquel ils aspirent.

La vieille femme d'aristocratie provinciale, riche et intraitable, sera tuée par Frantz, qui se justifiera de ce qu'elle est un être absolument inutile, qui ne vit que pour faire souffrir les autres. Elle passe ses jours enfermée dans sa chambre, entourée de tapisseries et d'armures ; elle se fait soigner par sa femme de chambre et quelques domestiques. Malgré quelques difficultés à dormir, elle jouit d'une robuste santé, ce qui rendra plus tard le meurtre violent et convulsif. Elle décide des moments graves de la vie, comme des petits rituels quotidiens, des mariages, comme de l'heure où tout le monde dans son château doit se coucher. Quand son univers calme, hostile, froid et orgueilleux, conservé dans sa riche maison, est dérangé par la fête du village, elle demande, en vain, le changement de date de son organisation, car elle est persuadée que le monde doit fonctionner d'après sa volonté : «Cette fête du pays est un supplice. J'ai voulu dix fois la faire mettre au mois d'août, pendant le temps de ma cure à Plombiers. Le maire prétend que c'est impossible, qu'elle est à cette date depuis le XII ${ }^{e}$ siècle. » (Anouilh 1970 : 77)

La Duchesse de Granat ne fait son apparition que dans le deuxième acte de la pièce, donc ce n'est pas à elle qu'appartient l'ouverture de l'œuvre. Durant le premier acte, les autres personnages ne cessent de parler d'elle ou de penser à elle, et quoique physiquement absente, elle devient aussi omniprésente dans la première partie de la pièce. Retenus par une panne de voiture hors de la maison et ne pouvant pas rentrer pour la nuit, Marie-Anne, la servante, 
Monime et Frantz pensent constamment à elle ; la pauvre Marie-Anne est horrifiée d'avance de sa réaction :

Marie-Anne : Madame ! Madame la Duchesse qui nous attend ! Déjà onze heures et demie ! C’est l'heure où nous devrions être rentrés... qu'allons-nous faire ? Que va penser Madame la Duchesse de cette équipée ?... C’est une équipée épouvantable ! Madame la Duchesse nous attend... Il faut que nous rentrions, Madame la duchesse ne me le pardonnerait pas... Madame la Duchesse ne voudra pas me recevoir demain... Ah ! J’appréhende notre retour ! À Granat, Madame la Duchesse exige que à onze heures tout le monde soit dans sa chambre... (Anouilh 1970 : 17-27)

Et quand ils lui adressent un appel téléphonique,

Marie-Anne : Bonsoir, Madame la Duchesse... mais Madame la Duchesse... mais, Madame la Duchesse... Oui, Madame la Duchesse... Monime : Je vous demande pardon, ma tante... Mais pourtant, ma tante.... Mais ma tante.... Si vous le voulez, ma tante... Je vous rassure ma tante... Oui, ma tante... Oui, ma tante... (Anouilh $1970: 23$ ),

La Duchesse de Granat ne laisse pas son interlocuteur finir sa phrase, comme c'était le cas avec la Duchesse de Brignoc; elle coupe la parole, surtout à la pauvre Marie-Anne, mais aussi à sa nièce Monime. Le personnage est physiquement absent de la scène, mais il est l'objet exclusif du discours, avant d'en devenir le sujet lors de son apparition dans le deuxième acte où, à la différence des autres personnages, elle prononce de longues tirades adressées à Frantz et à Monime. L'entrée de la Duchesse de Granat dans la salle, décrite dans les didascalies, évoque celle d'un roi parmi ses vassaux : «La duchesse, sorte de personnage fabuleux, entre, suivie d'Urbain, le maître d'hôtel, portant une couverture de fourrure... Elle s'installe dans son immense fauteuil à oreilles. Cérémonie lente et minutieuse » (Anouilh 1931 : 69). La fourrure qu'elle porte comme accessoire principal, l'hermine, suggère aussi bien le vêtement des cours de justice, blancheur au dessus de tout soupçon du Col d'Hermine, que le blason de Bretagne et sa devise " plutôt la mort que la souillure ", thème de la pièce, la pureté, la souillure, la justice.

La communication entre la Duchesse et Frantz est impossible. Comme l'explique Maurice Baudot, " chacun reste enfermé en lui-même, avec ses pensées et ses paroles qui flottent autour de lui comme un brouillard dont l'autre ne perçoit que l'aspect extérieur " (Baudot 1977 : 120). Elle cherche pour Frantz un parti convenant à sa modeste position sociale, alors que sa nièce doit épouser un noble de lignée aristocratique. Quand elle comprend que les jeunes gens sont amoureux l'un de l'autre, sa dureté s'accroît : lorsque le jeune homme lui avoue son sentiment pour Momine et la supplie de l'accepter, elle l'humilie, se moque de lui, ce qui fait basculer Frantz dans l'idée du meurtre. La fin abominable de la Duchesse est méritée, selon le jeune criminel, pauvre et amoureux, par l'étrange raison qu'il « [Frantz] la tue, en vertu du double droit qui, selon lui légitime le meurtre : celui d'aimer et celui de réussir. » (Vier 1976 : 27)

Le mécanisme du destin de la Duchesse se tend lorsque Philippe, journaliste auprès du tribunal, raconte à son ami Frantz l'histoire d'un meurtre qui préfigure ce qui va arriver : deux garçons ont tué leur grand-mère pour avoir de l'argent et se payer un voyage à Paris. Frantz s'intéresse vivement à leur sujet, et questionne Philippe sur leur motivation, le châtiment qu'ils encourent 
et apprend que, devant le tribunal les deux garçons se sont justifiés en déclarant qu'« [...] une vieille femme, cela ne sert à rien. » (Anouilh 1931 : 48). Une deuxième allusion génératrice de ce qui vient surgit dans les paroles de Florentine, une amie de Frantz et de Monime :

Florentine : Vous ne venez pas à Vichy avec moi ?

Monime : Tu sais bien que nous ne pouvons pas. Tante nous le défend.

Florentine : Comment ? Pas encore morte, cette vieille dame ? Elle manque vraiment d'esprit d'à-propos. Dites-lui de se dépêcher, que c'est beaucoup trop long. (Anouilh $1970: 67$ )

Ainsi, Frantz reçoit deux incitations au meurtre, l'une sérieuse, l'autre sarcastique, qui le poussent à agir pour une solution simple et définitive à la situation insupportable dans laquelle il prétend se trouver, le meurtre de la vieille duchesse. Tandis que dans Humulus le muet, la Duchesse, ordonnatrice du monde, était plutôt comique ou grotesque, elle est maléfique dans l'Hermine où, animée par le même désir d'assigner les rôles et les répliques d'un monde bien ordonné, elle sème le malheur autour d'elle et provoque ainsi sa propre fin.

\section{Lady Hurf dans Le bal des voleurs : le jeu comme évasion de l'ennui et du désespoir}

La Duchesse paraît pour la troisième fois dans Le bal des voleurs. Dans cette comédie-ballet l'auteur joue avec les personnages en les redoublant de masques et de déguisements. Les quiproquos, les malentendus et le jeu de masques se succèdent en cascade. ${ }^{3}$ Un trio de voleurs - Peterbono, Hector et le jeune Gustave - «travaille » à Vichy, une ville balnéaire. Par hasard, alors que le trio travaille sous le masque de nobles espagnols, ils font connaissance de Lady Hurf et de sa famille, ses deux nièces Éva et Juliette, et du vieil ami Lord Edgar. La Duchesse s'ennuie, et pour créer de l'imprévu, elle feint de reconnaître dans les voleurs déguisés des amis aristocrates espagnols qu'elle a connus à Biarritz, trente ans auparavant. Le plus jeune voleur, Gustave, est tombé amoureux d'Isabelle quand Isabelle, sans le savoir clairement, est tombée amoureuse de Gustave, et peut-être Éva estelle tombée amoureuse d'Hector. La Duchesse invite les voleurs déguisés en Espagnols dans sa maison, et un duo de banquiers, Dupont-Dufort père et fils, s'incruste, à l'affût de la dot des nièces. La situation cocasse du bal des voleurs est une pantomime dont elle tient les ficelles, une distraction de classe aristocratique, prise sur le compte du petit peuple prévisible des voleurs, mais bien davantage sur celui des vrais voleurs, les banquiers bourgeois en quête d'alliance et de dot. Elle invite tout son monde à participer à une soirée déguisée au Casino de Vichy, sur le thème désormais équivoque du bal des voleurs, prétend-elle, et s'amuse d'une situation dans laquelle le trio de voleurs déguisés, doit se trouver un déguisement de voleurs, ce qui " professionnellement », lui est impossible. Par contre, les banquiers n'éprouvent aucune difficulté à se déguiser en voleurs de comédie, en « apaches» selon le pittoresque de convention. Toute la troupe est vertement renvoyée du Casino car le bal était un bal des fleurs, - fâcheuse confusion de la Duchesse - et se retrouve dans son salon,

3 Paul Ginestier parle de la « perfection de l'invraisemblance » et souligne qu'il n'y a « rien de vrai dans cette histoire même pas le titre » (Ginestier $1969: 19)$. 
où se poursuit le joyeux quadrille du bal des voleurs, on ne peut dire plus justement, la. La Duchesse a le sens de l'intrigue et le mot juste : quiproquo de banquiers, voleurs professionnels mais un peu niais, et de voleurs de naissance, bons à rien. Le jeu cesse pour la Duchesse quand elle découvre l'amour naissant de sa nièce. Le dénouement se fait à la satisfaction de tous les protagonistes ou presque, car ce sont les banquiers qui, à cause de leur trop beau déguisement, sont emmenés par la police qu'ils ont eux-mêmes appelée. C'est pourquoi on considère cette œuvre comme la seule vraie pièce comique dans l'univers théâtral de Jean Anouilh. ${ }^{4}$

L'œuvre est divisée en quatre tableaux. La Duchesse, Lady Hurf, apparaît au milieu du premier tableau, se montre sur scène dans chaque acte, mais le nombre de ses sorties est considérablement réduit par rapport aux pièces précédentes, ainsi que la longueur de ses répliques. Les didascalies ne donnent aucune précision sur le personnage, ni sur son visage, ni sur ses traits de caractère. À la différence des deux cas précédents, Lady Hurf ne s'impose pas dans les échanges, la fréquence de ses apparitions ne dépasse pas celle des autres personnages et sur ce point, elle ne se distingue pas d'eux. Elle renonce, d'avance, à toute action et tout pouvoir contre les intrigues des uns et des autres.

Ses paroles dénotent un personnage résigné, fatigué et ennuyé par la vie, une vieille femme qui a tout obtenu, la richesse, la famille, les amants, les amis et les faux amis parasites, mais qui se sent vide et ressent sa solitude; elle a tout, mais rien qui la contente : "Je m'ennuie comme une vieille tapisserie. [...] J'ai cru pendant soixante ans qu'il fallait prendre la vie au sérieux. C’est beaucoup trop. Je suis d'humeur à faire une grande folie», avoue-t-elle (Anouilh 1963 : 24). Elle a tout, en somme, ce qui fait le bonheur du théâtre et de la vie. Si son « excentricité et [son] extravagance [sont] à base d'ennui et même de désespoir» (Marcel 1977 : 142), il ne lui reste plus rien, sauf une irrépressible envie de rire et de susciter de l'imprévu.

Dans son dialogue avec Éva, la nièce qui déjà lui ressemble, elle exprime sa déception devant la vie, mais aussi son attente, ou son espoir qu'elle place en la petite Juliette :

Lady Hurf: J'ai eu tout ce qu'une femme peut raisonnablement et même déraisonnablement souhaiter, l'argent, la puissance, les amants. Maintenant que je suis vieille je me retrouve autour de mes os aussi seule que lorsque j'étais une petite fille... et ce qui est plus grave, je me rends compte qu'entre cette petite fille et cette vieille femme il n'y a eu, avec beaucoup de bruit, qu'une solitude pire encore. Éva : Je vous croyais heureuse.

Lady Hurf: Tu n'as pas de bons yeux. Je joue un rôle. Je le joue bien comme tout ce que je fais, voilà tout. Toi, tu joues mal le tien! Elle lui caresse les cheveux. Petite fille, petite fille, vous serez toujours poursuivie par des désirs qui changeront de barbes sans que vous osiez jamais leur dire d'en garder une pour les aimer. Ma petite Juliette, elle, sera sauvée parce qu'elle est romanesque et simple. C'est une grâce qui n'est pas donnée à toutes...

(Anouilh $1963: 37$ )

La didascalie (elle lui caresse les cheveux), le ton de la duchesse et ses mots montrent une certaine tendresse pour ses nièces et pour la vie, celle qu'elle n’a pas vécue, mais veut encore

4 Pour en savoir plus, voire aussi notre article « Pour une lecture bergsonienne du Bal des voleurs de Jean Anouilh » in Romanica olomucensia (Voždová - Matoušková 2018 : 333-345). 
tenter de vivre. Elle décrit ce que sera ce bal des voleurs, puisqu'il se déroule ensuite conformément à ses paroles, Hector poursuit Éva de toutes ses barbes et déguisements sans retrouver son amour, et annonce le dénouement heureux pour Juliette ; la vie triomphe dans la joie romanesque et simple de la jeune fille. Et la mélancolie dans celle d'Éva. Juliette, Éva, deux pour une, c'est la duchesse Lady Hurf. La vie est un grand théâtre, elle aimerait pour un moment en être l'auteur, ou le régisseur. Ce qu'elle dit à Éva est comme une annonce, une sorte de prophétie, il s'agit pour elle non de se rassurer sur sa place dans la société, mais de voir bouger les gens et observer leurs actes ${ }^{5}$, de créer de l'imprévu, tenter de rire, afin que du mensonge du théâtre naisse une vérité, que la vie véritablement vécue soit celle dont Juliette porte l'espoir, mais qu'on ne peut y croire sans mélancolie.

À ses deux nièces, Lady Hurf laisse toute liberté, elle ne leur impose pas sa volonté. Pourtant, au fond d'elle-même, elle garde toujours la même obsession de diriger les gens, de dominer et décider de leur personnage, comme c'est le cas pour l'oncle Edgard, le vieux parent. Elle lui donne constamment des ordres, et des contrordres, l'accable de recommandations, tente de mener ses pas, et de ne jamais lui laisser formuler sa pensée. Il en a l'habitude et lit le Times. Elle a cependant beaucoup de mal à empêcher le vieil homme naïf, et aveugle à l'intrigue qu'elle mène, de dévoiler qu'il sait, comme elle sait elle-même et à bon droit, que le noble espagnol de Biarritz est mort il y a trente ans... dans les bras de la Duchesse (ou presque, dit-elle) et que les voleurs déguisés sont donc des imposteurs. Comme Lord Edgard ne comprend rien à ce qui se passe, et que la Duchesse le convainc qu'il se trompe, le jeu peut continuer jusqu'au dénouement.

La Duchesse du Bal des voleurs est beaucoup moins froide et orgueilleuse que ses doubles des pièces précédentes. Son approche des gens, de ses nièces en particulier, est fondée sur une indifférence sentimentale apparente. Son unique exigence dans les relations humaines, est le respect des conventions propres au rang et à la classe sociale ; elle joue à l'aristocrate avec les voleurs qui s'en tirent assez bien. Elle méprise par contre les banquiers et la bourgeoisie qu'ils représentent. Après un court moment de résistance, elle se laisse très vite charmer par l'amour naissant entre sa nièce Juliette et le jeune voleur Gustave. Elle-même a été incapable d'aimer, tout comme sa jeune incarnation, sa nièce Éva, par excès de sérieux et d'aspiration infinie, elle s'émeut de la passion de la romanesque et simple Juliette. Celle-ci a le sens simple de l'instant et du réel qu'il faut saisir. Lady Hurf tombe dans une sorte d'adoration pour cet amour, au point qu'elle approuve les jeunes gens, passant outre toutes les conventions sociales, et se réjouit mélancoliquement de leur bonheur. Le rire et la comédie qu'elle a suscités par ingéniosité a produit une vérité, les masques sont tombés laissant voir le visage nu des jeunes amoureux. La fantaisie et le hasard de ses intrigues ont engendré une sorte de mécanisme théâtral, la plus heureuse de ces intrigues étant de nommer le fait lui-même qui se déroule dans son salon, un bal des voleurs, puisqu'il rassemble en un joyeux quadrille, vrais banquiers et faux Espagnols, tous voleurs à leur manière. Le théâtre est un pur jeu de l'esprit, répète Anouilh, et la Duchesse qu'il s'invente porte son masque, accomplit comme auteur de la comédie un dévoilement de la vérité par l'enchaînement de sa propre prophétie, Duchesse pleine de rires et désormais, de sereine mélancolie.

5 On ne peut ne pas penser aux propos prononcés par Jean Anouilh à l'adresse de son grand maître Molière et à son attitude envers les gens et ses héros. 


\section{Madame Dupont-Dufort dans Le voyageur sans bagage : orgueil, sottise et hypocrisie}

Dans la pièce en cinq actes Le voyageur sans bagage, créée en 1936, Jean Anouilh introduit sur scène avec Madame Dupont-Dufort, une autre variante de la vieille duchesse. Le personnage fait son entrée dès le premier tableau, puis disparaît, et revient dans le cinquième : il ouvre et clôt la pièce. En tant que Duchesse, son rôle consiste à conduire les pas de son entourage, à parler la première et couper la parole à tous. Elle a une seule faiblesse, son inclination pour son neveu, le docteur Albert Jibelin, dont elle vante constamment les activités médicales et les succès.

Les didascalies ne donnent aucun détail précis concernant son portrait physique. L'important est son rôle joué dans la tragédie de Gaston, le "voyageur sans bagage » : Gaston est un amnésique, blessé pendant une bataille de la Première Guerre mondiale, trouvé devant un train de prisonniers venant d'Allemagne. Il ne se souvient pas de son passé, et passe quinze ans dans un asile, tranquille, sous le régime du Docteur Bonfant, tranquille en dehors des visites, le lundi : des familles essaient de le reconnaître comme l'un des leurs, des familles qui cherchent un fils disparu, par amour inconsolable ou par intérêt - Gaston a accumulé une coquette somme avec ses pensions de guerre. Mais depuis trois mois, le Docteur Bonfant a été remplacé par le Docteur Jibelin qui tente de rendre la mémoire au malheureux Gaston. Ce dernier est donc soumis aux expériences du Docteur Jibelin et aux entreprises de la Duchesse :

La Duchesse : C'est un grand psychiatre... que mon petit Albert... Confrontations, expertises graphologiques, analyses chimiques, enquêtes policières, rien de ce qui est humainement possible ne sera épargné pour que son malade trouve les siens. Côté clinique également... Songez qu'il a fait déjà dix-sept abcès de fixation!...C’est énorme et extrêmement courageux de la part de mon petit Albert. Car il faut bien le dire : c'est risqué.

Huspar : Mais Gaston?

La duchesse : De quoi pourrait-il se plaindre ? Tout est pour son bien. Il aura le derrière comme une écumoire sans doute, mais il retrouvera son passé. Et notre passé, c'est le meilleur de nousmêmes. Quel homme de cœur hésiterait entre son passé et la peau de son derrière ? (Anouilh $1970: 288)$

La Duchesse s'invente bienfaitrice de cet homme amnésique, veut le sauver et s'enivre de ce qu'elle fait pour lui. Mais en quelques phrases elle dévoile l'étendue drôlatique de sa bêtise et de son conformisme. À la fois, elle se laisse séduire par l'intérêt que portent au cas de son amnésique la presse et la société. Jouant la grande patriote, elle ne peut néanmoins se débarrasser de ses préjugés sociaux. Dans la même perspective de classe, quand elle recherche une famille pour Gaston, et voyage avec lui en compagnie de l'avocat Huspar, chargé des intérêts de Gaston, elle l'amène d'abord dans la riche bourgeoisie provinciale, chez les Renaud, et laisse de côté les familles pauvres situées plus bas sur l'échelle sociale.

Par sottise, la Duchesse se montre d'une dureté incroyable envers Gaston et son amnésie. Elle donne voix au conformisme social et familial qui ne peut accepter l'existence d'un homme sans passé et ne le laisse pas vivre. Désormais sous le joug de la patronnesse et du Docteur Jibelin, Gaston l'amnésique est traité comme un objet d'expérimentation ou un animal de laboratoire. 
À l'asile il a pu vivre tranquille, pendant des années entières, mais maintenant la Duchesse veut lui imposer une famille et un passé, alors qu'il semble transformé en un être indifférent à son sort, comme le Meursault de l'Étranger d'Albert Camus. Mais la Duchesse ne comprend rien de tout cela et voudrait, en vain, le voir ému par son destin présumé. Elle veut faire de lui, à l'aide des médias, un exemple à montrer, une histoire à faire pleurer le monde. Il ne peut opposer à tout cela qu'une sagesse sereine et indifférente, dont peu à peu on découvre la profondeur :

La Duchesse : Eh bien, Gaston, vous êtes ému, j’espère ? ...Il faut me soutenir,... et lui faire comprendre, qu'il doit être ému. ... Êtes-vous de pierre ? ...avez-vous le cœur plus dur que le roc ?... votre conduite laisserait croire que vous êtes un homme de marbre. Savez-vous ce que c'est du marbre ?... La pierre la plus dure. ... Vous êtes un des cas les plus troublants de la psychiatrie, une des énigmes les plus angoissantes de la grande guerre... vous êtes... le soldat inconnu vivant ... (Anouilh 1970 : 280-283)

Gaston l'amnésique découvre par l'effet d'anamnèse de certains détails qu'il est Jacques Renaud, fils de cette famille. Mais il refuse ce passé tant il lui déplaît d'être cet individu dont l'histoire est sombre et laide ; il découvre son passé, celui d'un enfant privé d'amour vrai par sa famille et qui s'est comporté comme un enfant perdu, stupide et violent. Il choisit l'existence de quelqu'un d'autre : il va devenir le neveu d'un petit enfant anglais, en inversant l'ordre du temps et des générations ; le plus jeune, l'enfant, est l'oncle du plus âgé, Gaston, qui comme lui a tout perdu, sa famille, ses parents, mais qui grâce à Gaston pourra retrouver ses biens. Ils partent ensemble pour une nouvelle vie, libres du passé. L'intrigue qui paraissait devoir beaucoup au Siegfried de Giraudoux, change radicalement de sens et de portée et ouvre une perspective morale, existentielle et métaphysique, alors que celle de Giraudoux était davantage dévouée à la réflexion historique.

Pour la description de son personnage, l'auteur ajoute de l'ironie et du sarcasme. Il n'en souligne plus la richesse et l'esprit dominateur, mais se concentre plutôt sur l'hypocrisie, la sottise et l'égoïsme extrêmes. En comparaison avec les variations de l'archétype précédentes de l'héroïne, dans la stratégie de la parole manquent aussi de longues tirades et diminue le nombre des phrases exclamatives et impératives. La présence quantitative affaiblie ne diminue aucunement son importance qualitative. La Duchesse du Voyageur sans bagage, contrairement à Gaston, est lourdement encombrée de ses idées toutes faites et des formules cocasses. En réalité, elle est plus sotte que méchante, d'ailleurs elle consent volontiers au choix que fait Gaston de sa nouvelle vie. Elle joue un rôle de contraste, ou de révélateur pour la sagesse réfléchie du Voyageur, car tout le poids de la convention sociale, des formules et des idées toutes faites, celle des histoires racontées d'avance, repose sur elle, mais le rire que suscitent ses propos produit un effet de vérité, de dévoilement. La liberté et la profondeur du sage amnésique ; amnésique d’abord par accident, ensuite par décision ; n'en ressort que plus intensément. En ce sens encore, quoique à contre sens de ses intentions, la Duchesse contribue à faire advenir une vérité du théâtre, le personnage n’est pas son passé, ce qu'est son histoire n'est pas décidé d'avance, le Voyageur est sans bagage. 


\section{La Duchesse Andinet d'Andaine dans Léocadia : manipulation ambiguë}

La cinquième incarnation de la Duchesse advient avec la pièce Léocadia, écrite en 1939. Albert, un jeune Prince, a vécu, deux ans auparavant, une brève mais intense histoire d'amour, une histoire de trois jours, avec une chanteuse excentrique, Léocadia, morte tragiquement, étranglée par son écharpe qu'elle avait nouée d'un trop grand geste. Après la mort de son aimée, le jeune Prince souffre et sombre dans la mélancolie. Comme il passe son temps à visiter les lieux où il a été heureux au cours des trois journées d'amour avec la Diva, sa Duchesse de tante, pour lui simplifier la vie dit-elle, achète tous les accessoires, choses et gens, qui forment le décor de l'histoire d'amour, afin de la reconstruire dans le parc de son château. Mais pour rejouer complètement cette brève histoire d'amour, il lui faut une Léocadia. Pour cela, elle tente d'engager Amanda à son service. Il s'agit d'une jeune ouvrière modiste, très belle, qui ressemble beaucoup à la cantatrice. Amanda accepte mystérieusement de jouer le rôle de la Diva pour trois jours. Ainsi l'histoire est mise en abyme par la Duchesse et par l'auteur, histoire jouée dans l'histoire, théâtre de la mémoire, du temps et de l'oubli, de la répétition et de la nouveauté, théâtre dans le théâtre pour quel dénouement?

Le personnage de la Duchesse est présent dans quatre des cinq actes de la pièce. L'acte quatrième est entièrement consacré à la rencontre intime d'Amanda et d'Albert; dans tous les autres actes, la Duchesse occupe le rôle central. Elle apparaît dès le début du premier acte, accompagnée de son cousin, le baron Hector. À la différence des pièces précédentes, l'auteur la décrit en détails dans les didascalies et, pour la première fois, Anouilh laisse son personnage se dévoiler aux autres quand il parle de lui-même, de sa famille, de son passé : la Duchesse est une vieille femme très riche, elle habite son château de Pont-au-Bronc, dans un immense parc, au point que, aussi loin que l'on marche, on n'en sort jamais... Elle a soixante-sept ans, mesure un mètre trente-huit, mais ne s'est jamais montrée sans talons; elle n'a plus de voix pour chanter, déteste la couleur verte et se vante constamment avec une emphase qui cache mal un secret humour, des exploits de ses ancêtres qui ont connu toute l'histoire de la France. Elle a l'habitude, tout en conversant avec les autres, de s'adresser à Gaston, son mari décédé. Chez elle, le passé se mêle sans cesse au présent et le réel à l'imaginaire.

Dans son boudoir de luxe, elle entre toujours avec des manières éclatantes, et attire l'attention de tous. Elle se présente en ces termes : " moi, une Andinet d’Andaine... J’ai connu le général Boulanger, les débuts de l'aviation, la fin du corset, les cheveux courts, la guerre mondiale... » (Anouilh 1939 : 169). Ses paroles sont d'un pathétique affecté, toujours cocasses, exagérées et drôles. Dans cette variante du rôle, la Duchesse paraît sensible et humaine, moins orgueilleuse que les précédentes. Elle exprime malgré tout sa volonté d’arranger la marche du monde selon ses plans, mais sous le signe de l'extravagance. C'est sa façon d'aimer, résume-t-elle. Elle dirige sa propriété, son château et son entourage en vraie patronne et règle fermement ses affaires. Toute sa vie tourne autour de l'affection qu'elle a pour Albert son «neveu », ou son " petit Albert». La Duchesse a pour Le Prince une affection intense et quelque peu exclusive. Elle le dit et le répète, elle l'aime beaucoup, elle l'adore, elle est prête à tout pour lui, pour le guérir de sa mélancolie. Mais on ne sait si sa force agissante prend sa source dans l'amour tendre pour le jeune homme ou dans son désir orgueilleux de continuer sa Maison, désir sous lequel elle dissimule parfois son inquiétude avec humour : 
Ce n'est vraiment pas la peine de s'être donné un mal de chien pour dominer la moitié du pays depuis le règne de Louis le Gros si l'on doit laisser sept cents ans plus tard son neveu baguenauder dans les rues avec les chauffeurs de taxi et des marchands de glace. (Anouilh 1939: 178)

Selon le même procédé de sincérité et de cocasserie, la Duchesse avoue sa sensibilité et se dit incapable de voir pleurer quelqu'un sans en éprouver du chagrin et sangloter à son tour. On ne sait à quoi s'en tenir de la sincérité ou du rôle, car aussitôt elle mêle comme d'habitude, par pudeur, l'aveu sincère et la bouffonnerie ; l'extravagance est son élément naturel. Ainsi, pour reconstituer son théâtre, elle achète le chauffeur de taxi avec sa machine, le marchand de glace et sa boutique, le garçon du bar avec l'auberge et ses musiciens tziganes, les bancs publics sur lesquels les amoureux se sont assis : ce fut le plus difficile car elle a dû faire un procès à la municipalité, mais elle l'a gagné. Elle se sert des gens comme d'objets, ils lui sont des accessoires, tels les statues, les tableaux et les meubles de sa propriété. Ainsi elle tente d'engager Amanda pour jouer le personnage de Léocadia : lorsqu'elle aperçoit par hasard la jeune femme dans un salon de modiste, elle découvre son incroyable ressemblance avec la chanteuse décédée. Dans son style hyperbolique, elle décrit Amanda, de concert avec Hector et le Maître d'Hôtel, comme « adorable, ravissante ", " hallucinante », " un petit Greuze qui se promène », " une fausse maigre de Boucher » (Anouilh 1939 : 189), bref, beaux compliments, qui montrent que, pour la Duchesse comme pour Hector et le Maître d'Hôtel, la beauté surpasse la condition sociale modeste et que les préjugés de caste n'ont pas cours chez elle.

La vieille dame voit avec le cœur, au-delà de toutes les apparences sociales, non pas seulement la beauté naturelle d'Amanda, mais l'incarnation d'un amour absolu auquel aspire le Prince. Le simulacre que la Duchesse a reconstruit dans le parc avec tous ses accessoires, choses et gens, se révélerait impuissant à guérir son neveu de sa mélancolie s’il n’y avait Amanda : résistant à un amour nouveau qui commence à naître, alors que tous deux jouent la scène de l'auberge, le Prince fanfaronne devant Amanda : «Ma tante est peut-être folle... une folle charmante, mais une folle, moi, j'ai beaucoup plus de bon sens qu'elle, croyez-le. » C'est Amanda qui, après s'être prêtée à ce jeu, commence à briser la convention sacrée de l'histoire d'amour, à prononcer d'autres répliques que les répliques prescrites, à commander d'autres boissons, à parler avec le jeune homme des choses de la vie quotidienne. Elle lutte contre la mélancolie du Prince et son fantôme de l'amour éternel, au nom de l'amour humain qu'elle incarne dans sa vaillance et sa simplicité. Mais le Prince, dans un dernier sursaut de défense, refuse son amour tout neuf pour Amanda et la jeune fille désespérée se réfugie sur un banc du jardin, où elle s'endort jusqu'à l'aurore. Quand la Duchesse au petit matin trouve Amanda, malheureuse dans le parc, elle prononce sa bouleversante prophétie :

(... La lumière a changé autour d'elles et les transformations vont s'accomplir à mesure que la duchesse parle comme une vieille fée) Le soleil est déjà presque levé. Tout espère et s'entrouvre au même rythme : les corolles, les jeunes feuilles hésitantes et les volets de bonnes gens. Sentez. Voici les premières bonnes odeurs du jour. L'odeur de la terre, l'odeur de l'herbe mouillée, puis celle du café qui est l'hommage de l'homme à l'aurore... Léocadia avait peut-être les forces de la nuit pour elle tout à l'heure... Vous avez vingt ans, vous êtes vivante et vous êtes amoureuse. Étirez vous au soleil et éclatez de rire. Toutes les jouissances du matin sont avec vous! (La Duchesse disparaît 
sans qu'on s'en aperçoive, le soleil est devenu tout d'un coup éclatant...) (Anouilh 1939 : 223)

La Duchesse disparaît pour faire place à l'amour naissant d'Albert et d'Amanda, l'amour réel, celui qui ne dure pas pour une éternité de trois jours, mais connaît les matins neufs, et l'odeur des cafés au lait que commande la simple et amoureuse Amanda pour elle-même et le Prince, assis à sa table, à elle.

L'auteur crée dans cette pièce une ambiance poétique qui cache une ironie profonde. Il donne l'image d'un grand amour perdu, mais d'après de courts propos clairsemés dans le texte, on comprend que Léocadia n’a pas vraiment aimé Albert, que ce n'était qu'un caprice de Diva, que Léocadia est une sorte de personnage de fiction s'exprimant dans une langue impossible faite de répliques, de rôles, de bouts d'opéras, qu'elle n'a jamais exprimé son amour à Albert dans les mots simples de l'amour, ni ne l'a appelé par son nom. L'histoire d'amour du Prince était une fiction. Par ses mises en scène qui redoublent la fiction, mais surtout par son regard du cœur qui lui rend Amanda visible, la Duchesse joue un rôle décisif dans la guérison du jeune homme, guérison d'un désir imaginaire, et se fait ainsi l'initiatrice et la protectrice d'un bonheur possible pour les jeunes gens dans le présent réel. La Duchesse est très sage en sa folie. La vieille fée annonciatrice, que décrit Anouilh dans la didascalie sous le masque, est la Fée du printemps. Si la Duchesse de Léocadia est fée, c'est parce qu'elle incarne, ironique et tendre, les pouvoirs magiques du théâtre. Léocadia était l'image composite de l'amour impossible et de ses romances confuses, que l'auteur a étranglé simplement d'un geste sec d'écharpe.

\section{Madame Desmermortes dans L'invitation au château : clairvoyance et bienfaisance}

Pour la dernière fois, le personnage de la Duchesse monte sur la scène d'Anouilh en 1946, dans L'invitation au château. Cette dernière incarnation montre une femme vieillie, rivée à son fauteuil roulant ; elle dépend de l'aide des autres. Propriétaire d'un château à la campagne, la Duchesse possède l'argent et le pouvoir sur sa famille, ses domestiques et ses deux neveux jumeaux, le cynique Horace et le timide et gentil Frédéric. Ce dernier croit tomber amoureux de Diana, la fille d'un riche financier juif, et veut se marier avec elle. Horace veut sauver son frère de ce mariage, il prépare ses intrigues et fait venir à l'occasion d'un bal au château la jeune danseuse Isabelle ; elle doit séduire Frédéric et ainsi empêcher le mariage. Mais le jeu produit un autre effet, Frédéric tombe amoureux d'Isabelle, Horace doit s'avouer que c'est lui qui aime Diana et cette inclination se dévoile.

Si la fréquence des sorties de la Duchesse diminue en comparaison des œuvres précédentes, leur importance reste indiscutable. Madame Desmermortes apparaît dans quatre des cinq actes de la pièce. Au premier acte, elle n'a qu'une entrée et une douzaine de répliques, mais elle en a une vingtaine (très courtes) dans le deuxième ; ce n'est que l'acte trois qui révèle son importance. Elle est absente du quatrième acte, surtout consacré à Isabelle mais joue un rôle primordial dans le dernier.

La duchesse vit au milieu de sa famille, mais ne se montre sur scène qu'accompagnée de mademoiselle Capulat, son aide et assistante, qui doit supporter ses remarques acerbes. Aucun défilé de domestiques ni de membres de la famille ne témoigne des liens de subordination ; ils sont 
néanmoins sous-entendus. Madame Desmermortes veut prendre le rôle du destin et diriger les vies de ses proches. C'est elle qui tient habituellement le rôle de dominatrice et le garde malgré les efforts de son neveu Horace. Bernard Beugnot, dans sa préface à une édition de l'œuvre, parle du « regard ironique, critique ou distancié de Madame Desmermortes qui parfois semble endosser la défroque des figurants du destin et des commentateurs ». (Beugnot 2012 : 12)

La caractérisation du personnage se limite cette fois-ci à quelques mots dans les didascalies : "Mme Desmermortes - poussée sur son fauteuil à roulettes par sa lectrice, Mlle Capulat..." (Anouilh 1945 : 48). En ce qui concerne son caractère, on peut le déduire encore une fois de ses actes, mais les traits habituels du personnage sont seulement esquissés et atténués. Elle a un fier sentiment de la tradition familiale, c'est pourquoi elle est furieuse d'apprendre que sa jeune nièce se laisse entretenir par un vieux et riche financier juif : "C'est monstrueux, c'est humiliant... Une Grandchamp ! Et par moi, une Desmermortes ! Si Antoine était encore de ce monde il en mourrait. » (Anouilh 1945 : 49) Elle s'inquiète du projet de mariage de Frédéric avec la riche Diana Messerschmann, car elle se rend bien compte de la nature faible de son neveu. Son ironie, son cynisme, mais aussi sa clairvoyance ressortent des paroles qu'elle adresse à l'intrigant Horace, et on comprend son plaisir à assister à ce grand théâtre qu'est le monde :

Avec toi, au moins, le sacrifice aurait eu du piquant. J'aime bien quand c'est le mouton qui risque de dévorer le grand prêtre. Avec lui, cela ne sera même pas drôle. Il sera affreusement mouton comme ton père. (Anouilh $1945: 50$ )

Madame Desmermortes révèle une lucidité extraordinaire et un grand talent d'observation, car sans en être informée, elle devine le manège d'Horace et déjoue ses plans. Ce dernier lui présente Isabelle comme la nièce de Romainville, ami de famille qui est invité au bal ; c'est pourquoi il conviendrait que la Duchesse envoie également son invitation à la jeune fille. La Duchesse interroge Romainville sur les origines et les parentés d'Isabelle. Jouissant d'une mémoire précise, elle découvre vite le mensonge. Quand, envahie par le doute sur la vérité de l'histoire que lui servent son neveu Horace et son ami Romainville, elle comprend la situation, elle refuse la mauvaise mise en scène inventée par Horace. Certes, elle est peut-être moins bavarde et plutôt silencieuse, mais d'autant plus attentive et réceptive.

Comme la Duchesse de Léocadia pour Amanda, Madame Desmermortes éprouve très vite une inclination pour la jeune fille modeste : «Elle a les yeux ravissants, Une biche aux abois. Un Greuze...». (Anouilh 1945 : 230) La Duchesse devine les qualités de la jeune fille, son innocence, sa franchise, sa tendresse et voit bien qu'à la différence de la gâtée Diana, elle formerait un couple heureux avec l'idéaliste Frédéric. Son désir de mettre en scène le jeu du monde, encore une fois, s'entend dans son soupir :

Je m'ennuie comme une vieille tapisserie. [...] Il faudrait que nous nous amusions aussi ce soir... Qu'est-ce que nous pourrions bien imaginer pour animer un peu cette soirée ? ... Le monde n'est plus drôle, il est temps de partir ! J’ai connu des bals inouïs de mon temps. » (Anouilh 1945 : 106)

Une situation inattendue lui offre tout de suite la possibilité de s'amuser, car la mère d'Isabelle, une musicienne sans talent et femme médiocre, arrive au château. Cela perturbe le plan d'Ho- 
race qui veut la cacher. En vain, la Capulat reconnaît en elle son amie de pensionnat, et la Duchesse l'introduit dans le jeu, en feignant de reconnaître en elle son ancienne amie, la Comtesse Funéla (comme c'était le cas de Lady Hurf avec les voleurs).

Dans le manège qu'Horace a imaginé, Isabelle doit se jeter à l'eau par amour pour Frédéric. Mais elle s'y jette pour son amour naissant pour Horace. La jeune fille est malheureuse et la Duchesse intervient, ne pouvant plus accepter de laisser souffrir cette jeune fille innocente :

Madame Desmermortes : Pourquoi avais-tu imaginé tout cela? ... Nous nous retrouverons là tous les deux, tout bêtes, sans être sûrs d'avoir très bien agi.

Horace : Vous m'étonnez ma tante. Je vous croyais beaucoup plus forte.

Madame Desmermortes : Figure-toi que moi aussi je me suis crue longtemps plus forte et j'en viens là.

Horace : La charité c'est de votre âge. Les vieilles dames de votre rang ont des pauvres, comme elles ont des demoiselles de compagnie et des petits chiens.

Madame Desmermortes : J'ai eu des pauvres bien entendu, toute ma vie, comme j'ai eu des chevaux et faces-à-main, On vit avec sa classe qu'on le veuille ou non, et, pour le courant, on fait comme les autres ou cela serait trop compliqué... C'est comme à ces tombolas où il y a trop de numéros et où on prend seulement une petite chance, sans y trop croire... (Anouilh 1945 : 217-221)

L'intervention autoritaire de la Duchesse change toute l'histoire et donne aux vies de tous les héros concernés un autre sens, il faut le souligner : un sens positif à leurs amours, leur bonheur et la plénitude de leur vie future. Le rôle de la duchesse dans cette fin est encore une fois décisif. De même, on ne peut pas ignorer la tendance à philosopher que le personnage commence à adopter.

\section{Pour caractériser l'archétype de la Duchesse}

" Le théâtre d'Anouilh parvient à être divers alors qu'il est composé d'un petit nombre d'éléments facilement repérables. C'est un théâtre expérimental et combinatoire ", écrit Christophe Mercier (Mercier 1995 : 65). Le personnage de la vieille Duchesse reste pour lui le plus typé de tous les personnages d'Anouilh. Il est repris six fois dans ses pièces, sur une période de presque vingt ans. Ce personnage-type est d'abord présenté sous un nom commun, «la duchesse », avec le nom de sa famille ou celui de ses propriétés qui l'individualisent, Duchesse de Brignoc, de Granat, de Lady Hurf, d'une d'Andinet d'Andaine, de Madame Dupont-Dufort ou de Madame Desmermortes. Dans les premières pièces (Humulus, Hermine) ses apparitions sont pathétiques et majestueuses ; la duchesse est présentée comme un personnage fabuleux, à la majesté parodique, signifiée par les objets, les armes, la fourrure. Elle est souvent accompagnée de domestiques et d'une suite. Dans les dernières pièces, ces attributs disparaissent pour faire place à une vieille femme, fatiguée par la vie mais très vivante et pleine d'esprit comme Lady Hurf, ou féerique comme La Duchesse de Léocadia. Dans le dernier cas, l'Invitation, elle est dépendante sur son fauteuil roulant mais n'a rien perdu de sa vivacité. Celle du Voyageur est un peu différente, bavarde, entreprenante, mais est un compendium de sottise, à l'image du monde bourgeois de l'après-guerre. 
Pour ce qui est du décor, des objets et accessoires qui entourent son personnage, l'auteur ne donne que de rares informations. L'ambiance reste toujours stylisée pour l'époque de 1900, ce qui devient le décor favori de Jean Anouilh. La ville de Vichy du Bal des Voleurs a gardé ses airs de ville fin de siècle 1900, mais la pièce se déroule en 1932. De même, le Voyageur est contemporain de son écriture, 1933/34. Le langage, les expressions, les allusions au contexte historique sont ceux des années d'écriture, dont les pièces sont contemporaines. Le contexte est signifié par les objets sociaux, le téléphone, le télégramme, la voiture, l’argent, etc. La duchesse vit dans sa propriété, accueille les invités dans un salon richement meublé, avec des tableaux précieux et de la porcelaine de valeur. Dans certaines pièces, on peut observer les détails concernant les vêtements, les bijoux et la richesse de la duchesse en général. L'espace où se situe l'histoire est souvent celui d'un château, entouré d'un parc, espace féerique quoique réel ou plausible dans Léocadia, ou bien d'une villa dans une station thermale.

Comme l'univers théâtral d'Anouilh se limite au cercle familial - Jacques Vier reprend l'image du roman de 1932 de François Mauriac, du Noud de vipères (Vier 1976 : 29), - la famille reste le lieu du drame. La Duchesse s'intègre dans les liens familiaux d'une manière particulière. Elle occupe dans sa famille la position de la grand-mère, de la tante ou de la protectrice de jeunes héros. Dans Humulus elle à un petit-fils, Humulus, dans l'Hermine une nièce, Monime, et un protégé, Frantz, dans Le bal deux nièces, Éva et Juliette, dans le Voyageur un protégé spirituel, Gaston, dans Léocadia un neveu, Albert, et enfin, dans L'Invitation deux neveux, Frédéric et Horatz, et une nièce, Dorothée. À part le nombre de domestiques, la duchesse se montre accompagnée par les amis de la famille ou par ses secrétaires, comme le gouverneur, l'oncle Hector (Humulus), le maître d'hôtel Urbain, la femme de chambre Marie-Anne (Hermine), le Lord Edgar (Le bal), l'avocat Huspar (Voyageur), le baron Hector et son mari Gaston, mort mais omniprésent (Léoca$\mathrm{dia}$ ) et la dame de compagnie mademoiselle Capulet (Invitation). Ils servent souvent de cible ou de témoins de la mauvaise humeur de la duchesse et de ses caprices. La duchesse, dans tous les cas, se comporte en chef de sa famille, et tous les autres protagonistes se définissent par rapport à elle. Elle ne représente jamais un personnage isolé et sort constamment accompagnée de la famille, d'un vieil ami ou membre de la famille, puis de ses jeunes nièces et protégés.

Quant à la présence de la Duchesse sur scène, à la fréquence de ses entrées et sorties, ou à la quantité et l'importance de ses discours, on peut constater que dans aucune pièce elle ne figure dans tous les actes. L'Hermine est un cas particulier, dans lequel elle est omniprésente même si elle est physiquement absente, ou morte, car tous les autres personnages ne cessent de parler d'elle ou de penser à elle. Si elle recourt au pathétique, son discours s'étire jusqu'à la tirade dans l'Hermine ou bien l'Invitation. Elle s'impose à son interlocuteur, donne ses opinions, pose des questions et dirige la discussion. Le degré de son écoute varie d'une pièce à l'autre : les duchesses de Humulus et de l'Hermine n'entendent pas l'autre, coupent la parole et leur conversation se présente comme une suite de monologues. La Duchesse du Voyageur n'écoute guère et bavarde un peu à tort et à travers, mais cela produit comme dans les autres cas un puissant effet théâtral. Les autres sont souvent très bavardes et fantaisistes, d'une écoute discrètement présente, parfois très intense comme dans Léocadia, le Bal des voleurs, et l'Invitation.

Le langage utilisé par les duchesses correspond aux modifications de leur comportement et aux changements de leur caractère. Dans Humulus, l'Hermine, Le voyageur la Duchesse s'exprime avec pathos et sa langue sert d'accessoire à son personnage, elle est assortie à sa position 
de grande dame des temps passés. L'effet en est encore accentué par une forme d'exagération poétique dans Léocadia, mais celle-ci joue délibérément avec les formes du langage et peut tenir tous les registres jusqu'à l'économie la plus intime. Seules Lady Hurf du Bal et Madame Desmermortes de L'Invitation ne se différencient pas, par leur façon de parler, des autres protagonistes.

L'auteur n'insiste pas sur le portrait physique de son personnage, mais la Duchesse d'Andinet d'Andaine se décrit elle-même en détail. Beaucoup plus important pour lui est son caractère. La Duchesse d'Humulus ne parle nullement d'elle-même, sauf pour dire brièvement qu'elle a ses pauvres. Celle de L'Hermine ne dit rien de personnel, comme la Duchesse Dupont-Dufort du Voyageur. Lady Hurf, du Bal, avoue son mécontentement de la vie, son manque d'amour et son désir de s'amuser dans sa vieillesse. Il en va de même pour Madame Desmermortes, de l'Invitation : elle aussi a ses pauvres, comme celle de Humulus, elle aussi s'ennuie et veut s'amuser comme Lady Hurf, du Bal.

Le personnage est unique : la Duchesse. Et beaucoup de traits sont communs. Or, il y a deux qualités de caractère qui se font omniprésentes dans toute les pièces et représentent les traits majeurs de la caractéristique du personnage anouilhien de la duchesse. Dans le personnage de la vieille duchesse, Anouilh a créé un être orgueilleux, dure et insensible. Toutes les héroïnes analysées sont avant tout égoïstes, indifférentes à leurs entourages, privées du sentiment de compassion pour les autres, même les plus proches, incapables d'aimer et de partager le sentiment. Elle ne connaissent ni pitié pour les souffrants ni tolérance pour la jeunesse. Même si elles possèdent une dose des expériences de la vie, elles refusent de la partager avec leurs proches. Si le personnage de la duchesse de Granat de l'Hermine va dans cette dureté et insensibilité jusqu'à une méchanceté extrême envers ses jeunes Monime et Frantz qui la remplit de plaisir cruel, celui de Madame Desmermortes de l'Invitation au château, créé quinze ans après, en est au contraire affaibli, capable de percevoir les inclinations de ses neveux et prêt à les comprendre. La duchesse se vante de ses origines aristocratiques, du passé de sa famille, de ses connaissances de la race noble et elle différencie les gens d'avance d'après leur classe sociale. La seule aristocratie qui compte pour elle est celle de la naissance, nullement celle du cœur et de l'esprit. L'hypocrisie extrême de ces figures féminines de la duchesse remonte à la surface notamment dans leur conduite envers les pauvres. Insensibles et cruelles, elles font de la charité, car c'est l'usage de leur classe sociale, comme si c'était une formalité de l'étiquette de la cour.

Le deuxième trait majeur de la figure de la duchesse chez Anouilh prend sa source dans son insensibilité et représente la volonté de domination. La duchesse semble vouloir être une sorte de grand horloger voltairien. Comme elle possède de la richesse matérielle, elle se sent toute-puissante. Il est vrai que les autres personnages dépendent financièrement d'elle, qu'il s'agisse de ses jeunes parents, de ses vieux amis et de gens de différents liens de parenté, et bien sûr de ses domestiques. En leur donnant la sécurité matérielle, elle leur impose ses règles de vie et exige la soumission absolue dans tous les domaines dont, pour les jeunes, le plus sensible, fragile et blessant, le domaine de l'amour. L'argent figure en tant qu'arme absolue de la duchesse ; au contraire la jeunesse, l'amour et l'ennui représentent ses ennemis. La duchesse se place dans le rôle du destin, joue avec les gens comme aux échecs, rien que pour sa simple distraction. La plus forte de ces figures est présentée par la duchesse de L'Hermine. Elle n'est pas capable de quitter cette attitude dominatrice, même devant la mort, et préfère plutôt mourir que de renoncer à sa résolution insensée et de laisser les jeunes vivre d'après leurs désirs. Donc ce rôle de dominatrice 
surmonte toutes les autres facultés, elle dépasse même les frontières de ce monde et continue de régner jusque dans la mort. Mais à l'opposé du spectre, D’Andinet D’Andaine est reine de la vie.

Thérèse Malachy, dans sa typologie des personnages de l'univers anouilhien, définit aussi celui de manipulateur. Il « se manifeste sous forme de personnages metteurs en scène - professionnels et amateurs - ou en la personne de l'auteur " (Malachy 1978 : 20). La duchesse représente alors dans sa perspective une incarnation parfaite de ce manipulateur. Jacques Vier, au contraire, probablement influencé par le premier théâtre d'Anouilh aux thèmes antiques, ce qui n'est à notre avis pas ce cas-là, voit un certain rôle libérateur de celle-ci. D’après lui, la duchesse appartient aux héros anouilhiens audacieux qui, «par leurs faits, et par leur volonté de mener le jeu, luttent contre les caprices du destin et veulent ainsi libérer les hommes de la prédestination et des dieux. » (Vier 1976 : 76) Alors, signe de fatalité de l'action comme dans L'Hermine ou messagère de liberté comme dans le Bal ou dans Léocadia, la Duchesse rappelle que l'auteur avance masqué, et renverse la question avec Pirandello, ce sont les personnages qui sont en quête d'auteur.

Avec la vieille duchesse, Jean Anouilh crée un type original d'héroïne dramatique qui intervient comme un déclencheur d'action dramatique dans les pièces où il apparaît. Pour certains critiques, le personnage d'Anouilh, en général, est l'élément d'une combinatoire, et reste plus ou moins le même avec de petites variantes, selon le contexte de la pièce. ${ }^{6}$ Or nous constatons que celui de la Duchesse évolue. De l'anti-héros et monstre de cauchemar, il se métamorphose en créature humaine. Ce changement s'effectue pendant une période de plus de quinze ans et accompagne l'évolution de l'auteur. Si, dans ses premières apparitions, la duchesse se montrait orgueilleuse, inhumaine et maléfique, avec les années, mûrie, vieillie, affolée ou allégée, selon les variantes, elle incarne celle qui aime surtout faire du théâtre et le faire jouer aux autres. Elle joue la folle pour ne pas penser à tout ce qu'elle a manqué dans la vie, pour oublier la réalité, pour s'amuser et vivre dans l'illusion théâtrale. En tant que meneuse de jeu, elle peut, grâce à cette illusion, faire triompher le bonheur et ainsi nier la cruauté de la vie réelle. Telle une marionnette, elle incarne, dans cet univers de commedia dell'arte, une caricature du personnage, mais de personnage qui dans la comédie, tire elle aussi les ficelles. Si l'auteur lui-même a avoué dans une de ses lettres qu'il était « la vieille duchesse, folle de ses personnages ", c'est en vertu de ce pouvoir des duchesses, en bien en mal, de donner vie aux personnages et, par l'illusion théâtrale, par le divertissement, rendre au spectateur une liberté d'esprit et un pouvoir accru de vivre. Dans ce sens-là on peut constater que la duchesse est le porte-parole de l'auteur et transmet sa philosophie.

6 Adélaïde Jacquemard-Truc souligne sa stylisation extrême de marionette. (Jacquemard-Truc 213 : 77). Malachy ne voit aucune évolution des personnages ; d’après elle il s'agit de types donnés et retravaillés constamment. "Si évolution il y a, c'est dans les retouches opérées sur les types existants, consistant à les vieillir ou à les jeter dans des situations dramatiques différentes, et surtout à leur faire changer de milieu social. Mais l'essentiel du personnage est conservé ». (Malachy 1975 : 28) 


\section{Références bibliographiques}

Anouilh, J. (1963). Le Bal des voleurs. In Théâtre complet de Jean Anouilh. Tome I (pp. 13-75). Lausanne: La Guilde du Livre.

. (1963). Humulus le Muet. In Théâtre complet de Jean Anouilh. Tome I (pp. 1-11). Lausanne: La Guilde du Livre.

. (1963). Léocadia. In Théâtre complet de Jean Anouilh. Tome I (pp. 161-231). Lausanne: La Guilde du Livre.

(1970). Hermine. In Pièces noires (pp. 9-131). Paris: La Table Ronde.

(1970). Le Voyageur sans bagages. In Pièces noires (pp. 277-386). Paris: La Table Ronde.

. (1987). La Vicomtesse d'Eristal n'a pas reçu son balai mécanique. Souvenirs d'un jeune homme.

Paris: La Table Ronde.

. (2012). L'invitation au château. In Théâtre complet de Jean Anouilh. Tome I (pp. 813-910). Lausanne: La Guilde du Livre.

Baudot, M. (1977). Pureté et aliénation. In B. Beugnot (Ed.), Les critiques de notre temps et Jean Anouilh (pp. 116-121). Paris: Garnier.

Beugnot, B. (2012). Préface. In J. Anouilh, L’invitation au château (pp. 7-27). Paris: Gallimard.

Ginestier, P. (1969), Jean Anouilh. Paris: Seghers.

Jacquemard-Truc, A. (2013). De l'influence de la dramaturgie de Musset dans les Pièces roses. In E. Le

Corre, \& B. Barut (Eds.), Jean Anouilh. Artisan du théâtre (pp. 71-82). Rennes: Presses universitaires de Rennes.

Malachy, T. (1978). Jean Anouilh, les Problèmes de l'existence dans un théâtre de marionettes. Paris: Nizet.

Marcel, G. (1977). La vision tragique. In B. Beugnot (Ed.), Les critiques de notre temps et Jean Anouilh (pp. 140-142). Paris : Garnier.

Mercier, Ch. (1995). Pour saluer Jean Anouilh. Paris: Bartillat.

Vier, J. (1976). Le théâtre de Jean Anouilh. Paris: CDU et SEDES.

Voždová, M.; \& Matoušková, J. (2018). Pour une lecture bergsonienne du Bal des voleurs de Jean Anouilh.

Romanica olomucensia, 30 (2), 333-345. 
